\title{
Heralding the Digitalization of Life in Post-Pandemic East Asian Societies
}

\author{
Calvin Wai-Loon Ho 1 - Karel Caals • \\ Haihong Zhang
}

Received: 2 May 2020 / Accepted: 21 September 2020

(C) Journal of Bioethical Inquiry Pty Ltd. 2020

\begin{abstract}
Following the outbreak of what would become the COVID-19 pandemic, social distancing measures were quickly introduced across East Asiaincluding drastic shelter-in-place orders in some cities - drawing on experience with the outbreak of severe acute respiratory syndrome (SARS) almost two decades ago. "Smart City" technologies and other digital tools were quickly deployed for infection control purposes, ranging from conventional thermal scanning cameras to digital tracing in the surveillance of at-risk individuals. Chatbots endowed with artificial intelligence have also been deployed to shift part of healthcare provision away from hospitals and to support a number of programmes for self-management of chronic disease in the community. With the closure of schools and adults working from home, digital technologies have also sustained many aspects of both professional and social life at a pace and scale not considered to be practicable before the outbreak. This paper considers
\end{abstract}

\author{
C. W.-L. Ho $(\bowtie)$ \\ Faculty of Law and Centre for Medical Ethics and Law, The \\ University of Hong Kong, Cheng Yu Tung Tower, Centennial \\ Campus, Pokfulam Road, Hong Kong S.A.R., People's Republic \\ of China \\ e-mail: cwlho@hku.hk \\ K. Caals \\ Centre for Biomedical Ethics, Yong Loo Lin School of Medicine, \\ National University of Singapore, Block MD11, Clinical Research \\ Centre \#02-03, 10 Medical Drive 117597, Singapore
}

H. Zhang

Health Science Center, Peking University, 38 Xueyuan Road, Haidian District, Beijing, People's Republic of China how these new experiences with digital technologies in public health surveillance are spurring digitalization in East Asian societies beyond the conventional public health context. It also considers some of the concerns and challenges that are likely to arise with rapid digitalization, particularly in healthcare.

Keywords COVID-19 . Contact tracing $\cdot$ Public health surveillance $\cdot$ Digital health $\cdot$ Mobile health $\cdot$ Artificial intelligence

Drawing on prior experience with the outbreak of severe acute respiratory syndrome (SARS) almost two decades ago, social distancing measures (including drastic shelter-in-place orders in some cities) were quickly introduced across China in the early phase of the COVID19 outbreak. Other East Asian societies followed suit, but unlike during the SARS outbreak, many public health measures for surveillance of at-risk individuals and infection control are now enhanced by "Smart City" and other digital technologies. Digital tools, including chatbots endowed with artificial intelligence (AI) (Miner, Larango, and Kocaballi 2020), have been deployed to shift part of healthcare provision away from hospitals and to support self-management of chronic disease in the community. With the closure of schools and adults working from home, digital technologies have sustained many aspects of professional and social life at a pace and scale not considered to be practicable before the outbreak. In this paper, we explain how the COVID-19 pandemic has mainstreamed digital 
technologies in public health surveillance, as they were previously only applied in limited and controlled settings, like health-related research. With the intensive use of digital technologies and resulting data amassed during the pandemic, these technologies will have a fundamental role in shaping the post-pandemic public health and healthcare landscapes (Goodman et al. 2020), as well as other aspects of social life, in these already increasingly digitalized East Asian societies. It is beyond the scope of this paper to delve into details of the digitalization of life. We seek only to show how the COVID-19 pandemic has anchored and spurred digitalization forward. By "digitalized" and "digitalization," we refer to the added value of applying digital technologies (such as data analytics, AI, and robotics) to interventions directed at meeting healthcare, public health and, even more broadly, social goals (Health Education England 2019). With rapid digitalization, the centrestaging of challenges with (public) health systems and data integration, data governance (including concerns about privacy and cybersecurity), and raising data literacy across all levels of society may well be one of the lasting legacies of this pandemic.

\section{Fast-Tracking Surveillance-Based Digital Technologies}

With the COVID-19 outbreak, digital tools like contact tracing applications have been quickly deployed for the purpose of infection control. At the epicentre of the outbreak, a subsidiary of China's biggest insurer, Ping An Insurance, announced that its AI screening system for the disease has been in operation since February 18, 2020 (Olano 2020). Within less than a month, this system has reportedly completed over 580,000 screenings in 47,000 households within seventeen communities in Wuhan and has successfully identified more than 1,600 individuals suspected to have contracted COVID-19. In essence, the insurer's screening system operated 3,000 AI chatbots working concurrently, with the capacity of screening a total of 1.5 million audio calls per day. Digital contact tracing tools were then applied to track residents suspected to be symptomatic and individuals who might have been infected by them. The application of digital technologies such as these may have helped to flatten the infection curve in mainland China and perhaps also lowered mortality as rapid diagnosis of COVID-19 allows early treatment (Ji et al. 2020). In Hong Kong, wristband-based smartphone-tracking technology has been deployed to ensure that quarantine orders were observed (Sharwood 2020). Similar digital tracing technologies have been applied in Taiwan and Singapore for contact-tracing purposes. The contact-tracing smartphone application that was developed by the Singaporean government has been adapted by the Japanese government, for the purpose of warning users who have been in contact with someone diagnosed with COVID-19 (Japan Times 2020). In South Korea, widespread testing and contact tracing through digital and other means enabled its health authorities to bring the number of infection (at one point the world's second highest after China) under control without having to lock down the country (COVID-19 National Emergency Response Center (Korea) 2020).

Where privacy concerns have arisen, some governments have been responsive. In Singapore, for example, a Bluetooth-based contact tracing token that was developed to address the technical limitations of "TraceTogether" does not have any location technology like GPS and does not connect to the Internet, which means that it cannot capture geolocation data and that the data cannot be extracted remotely. These features were developed in response to privacy concerns expressed by some citizens in an online petition (Baharudin 2020; Mahmud 2020). In effect, when someone tests positive for COVID-19, public health workers will have to collect the patient's token to download the proximity data from the device, in order to determine the close contacts of the infected person. As further assurance, data is stored on the token in an encrypted format for no more than twenty-five days. Additionally, smart Internet of things (IoT) technology has been developed to ensure that the social distance requirement of one metre between persons is observed. This technology, called smart distancing sensors on premise (SDSP), uses ceiling sensors to measure the distance between individuals (Yau 2020). If two persons are less than a metre apart, a laser signal will be triggered to alert customers and staff on the premises (such as a restaurant or in the airport) that a safe distance needs to be restored. Applied separately, the contact tracing tokens and SDSP technology are unlikely to raise serious privacy concerns. It is less clear if their combined use to 
collect data about individuals on an ongoing basis will rekindle these concerns.

\section{Extending the Public Health Agenda}

As East Asian societies learn to live with the pandemic, digital technologies will be adapted and deployed to address a wider range of public health challenges, including management of non-communicable (or chronic) diseases, control of behavioural risk factors, and more intricate monitoring of healthcare services. While this technological trajectory might have already been embedded in the health policies of these societies increasingly oriented towards health promotion, disease prevention, and adoption of healthy behaviours, there is little doubt that the pandemic has spurred the technological agenda forward.

An enduring impact of the pandemic is in the digitalization of healthcare as telemedicine services and of health insurance, with insurers assuming a more active role in monitoring and evaluating health and healthcare services (Ho, Ali, and Caals 2020). Demand in telemedicine services delivered through digital platforms grew as lockdowns proliferated and social distancing measures reduced access to hospitals except for those with COVID-19 or other emergencies. The newly acquired familiarity with virtual consultations on telemedicine platforms has helped patients overcome initial behavioural and economic barriers to the adoption of telemedicine. A healthcare survey indicated a nearly 900 per cent increase in the number of new users on a Chinese healthcare services platform (Ping An Good Doctor), with similar trends being reported for an online platform for healthcare providers (Ding Xiang Yuan) and a telemedicine platform (Chunyu Doctor) in China (Kapur and Boulton 2020). There has also been a surge in usage of digital health platforms in Singapore (e.g., MyDoc), and various initiatives have been introduced in South Korea and Japan to promote telemedicine services.

Across East Asian societies, the use of surveillancebased digital technologies has also extended well beyond public health and other activities that relate directly to infection control. The digitalization of work is becoming evident, as occupational and public health considerations fundamentally reconfigure office spaces, programmes, schedules, and practices. Companies have adapted and applied smartphone applications to determine if their employees are healthy enough to be at the worksite. Digital technologies enable work-from-home and work-in-shifts arrangements as social distancing requirements limit the number of people who are able to share work and work-related spaces, from open floor plans to elevators. A degree of digital surveillance is also applied in platforms for conducting virtual meetings and online training courses, whether within an academic or work setting. This technological feature enabled Zoom, which provides a cloud-based peer-topeer software platform for teleconferencing, to block certain users from joining video calls (Yang 2020). Despite concerns over surveillance and censorship, such software platforms will become much more entrenched, as they enjoy a substantial cost advantage over traditional classroom teaching.

On the policy front, perceived success in the deployment of digital technology to control the transmission of COVID-19 will help legitimize substantial resources committed by East Asian governments to build new digital infrastructure, aside from this being part of a broader effort to initiate economic recovery. In China, State Grid Corporation will invest 24.7 billion yuan (or about USD 3.5 billion) in 2020 in digital infrastructure, in cooperation with Chinese technology giants Tencent, Alibaba, and Huawei (Chen 2020). This new digital infrastructure, comprising big data centres, $5 \mathrm{G}$ networks, and artificial intelligence, is expected to profoundly reshape various sectors including healthcare. Digitalization could generate significant gains, but it is as yet unclear how anticipated concerns will be addressed.

\section{Coping with Digitalization}

Important technical limitations will need to be surmounted, as recent tests of $5 \mathrm{G}$ network speeds and coverage in Seoul, Beijing, Tokyo, and Hong Kong have produced mixed results (Bloomberg News 2020). It is also not clear how exactly digital solutions will be applied in East Asian societies to support post-pandemic public health action, including the monitoring and control of infectious diseases. In the aftermath of the pandemic, it is likely that strategies for public health digitalization will be drawn up to build on experiences gained from pervasive use of digital tools throughout the course of the outbreak. The rate of digitalization is also likely to increase as very few aspects of social life (ranging from employment to entertainment) are 
untouched by digital technologies. However, digitalization (particularly for surveillance-related purposes) does not alter ethical, legal, and social concerns and may even aggravate them. For instance, the transactional nature of virtual meetings or the use of software to monitor time spent at work by employees via their laptops could become a source of anxiety. To advance health systems and public health goals in an optimal manner, surveillance and other digital health measures must occur within a robust governance framework (McKee, van Schalkwyk, and Stuckler 2019; Ricciardi et al. 2019). Where digital contact tracing and surveillance are concerned, instructive ethical guidance is available (see, for instance, Kahn and Johns Hopkins Project 2020; World Health Organization 2020; UNICEF 2020). However, their implementation in policy, regulation, and governance across the different East Asian societies will be a key challenge in the foreseeable future (Bustreo and Tanner 2020).

The databases resulting from current digital efforts to contain the pandemic will need to be integrated into growing health and public health data infrastructures, while anticipating further use of digital technology and health data collection. As the pandemic continues to identify weaknesses within healthcare systems around the world, digital tools and data collection must be harnessed for the benefit of the vulnerable or marginalized, in particular. In this vein, the contact tracing token used in Singapore was in part developed with older people in mind, particularly those who lack social support and do not own or use digital devices. Even then, appropriate ethical and legal safeguards (such as data protection and privacy laws), inclusive communication strategies, and a degree of trust in the government must be in place (World Health Organization 2020). Equally, digital technology can support preventive and primary care, for example via telehealth and mobile health, thereby helping to shift healthcare in many East Asian health systems away from a heavy focus on tertiary care. Perhaps most critically, current and future public health surveillance, whether with the benefit of digitalization or not, must be sustained or undertaken within an appropriate oversight or governance framework to evaluate effectiveness and ensure that ethical (as well as legal and social) challenges are anticipated and addressed systematically and transparently (World Health Organization 2017, 13). As this pandemic makes clear, safeguards against discrimination and cybersecurity risks, together with measures to promote appropriate data stewardship and greater digital literacy (inclusive of data rights and responsibilities) across all levels of society are needed to build and sustain trustworthiness and credibility.

\section{Conclusion}

New experiences with surveillance-based digital technologies are likely to be among the most important lessons learnt through this pandemic for East Asian societies. Widely perceived to have helped contain and manage the COVID-19 outbreak, digitalization is already moving beyond public health interventions. In the foreseeable future, digitalization of healthcare and of work itself, among many other aspects of social life, is expected to accelerate. Its impact on social organization, practices, and expectations will be profound, but digital technologies are also responsive to normative and social forces. While much has already been written about the ethical concerns and challenges that have arisen or are likely to arise, it is less clear how they will be addressed across East Asia. For a start, it is imperative for public dialogue to be initiated in order to inform political and collective choices and to secure legitimacy that underscores trust. While substantial economic resources are already committed to advancing the digital agenda, an equal or greater investment of ethical and legal resources is needed to chart and govern a collective digital future that is responsible, equitable, and sustainable.

\section{References}

Baharudin, H. 2020. Covid-19 contact tracing device will not be an electronic tag, to be rolled out in June. The Straits Times, June 8. http://www.straitstimes.com.sg/singapore/covid-19contact-tracing-device-will-not-be-an-electronic-tag-to-berolled-out-in-june. Accessed June 30, 2020.

Bloomberg News. 2020. We tested 5G networks across Asian cities. The Verdict: Patchy. Bloomberg News, June 14. https:/www.bloomberg.com/news/articles/2020-06-13 /world-s-first-5g-networks-are-still-more-patchy-thanpowerful. Accessed June 30, 2020.

Bustreo, F., and M. Tanner. 2020. How do we reimagine health in a digital age? Bulletin of the World Health Organization 98(4): 232.

Chen, C. 2020. State Grid plans big increase in digital infrastructure use. South China Morning Post, June 17, at B4.

COVID-19 National Emergency Response Center, Epidemiology $\&$ Case Management Team, Korea Centers for Disease 
Control \& Prevention. 2020. Contact transmission of COVID-19 in South Korea: Novel investigation techniques for tracing contacts. Osong Public Health and Research Perspectives 11(1): 60-63.

Goodman, K., D. Zandi, A. Reis, and E. Vayena. 2020. Balancing risks and benefits of artificial intelligence in the health sector. Bulletin of the World Health Organization 98(4): 230-230A.

Health Education England. 2019. The Topol Review: Preparing the healthcare workforce to deliver the digital future. https://topol.hee.nhs.uk. Accessed June 30, 2020.

Ho, C.W-L., J. Ali, and K. Caals. 2020. Ensuring trustworthy use of artificial intelligence and big data analytics in health insurance. Bulletin of the World Health Organization 98(4): 263-269.

Japan Times. 2020. Japanese government to release coronavirus contact-tracing app in May. Japan Times, April 29, 2020. https://www.japantimes.co.jp/news/2020/04/29 /national/japanese-government-release-coronavirus-contacttracing-app-may/\#.XqzR4kBuIRk. Accessed May 1, 2020.

Ji, Y., Z. Ma, M.P. Peppelenbosch, and Q. Pan. 2020. Potential association between Covid-19 mortality and health-care resource availability. The Lancet Global Health 8(4): E480.

Kahn, J., and Johns Hopkins Project on Ethics and Governance of Digital Contact Tracing Technologies. 2020. Digital contact tracing for pandemic response. Baltimore: Johns Hopkins University Press.

Kapur, V., and A. Boulton. 2020. Covid-19 accelerates the adoption of telemedicine in Asia-Pacific countries. Bain \& Company, April 27, 2020. https://www.bain. com/insights/covid-19-accelerates-the-adoption-oftelemedicine-in-asia-pacific-countries/. Accessed June 30, 2020.

Mahmud, A.H. 2020. COVID-19: Govt developing wearable contact tracing device, may be distributed to everyone in Singapore. Channel News Asia, June 5, 2020. https://www. channelnewsasia.com/news/singapore/covid-19-contacttracing-device-trace-together-app-12806842. Accessed June 30, 2020.

McKee, M., M.C.I. van Schalkwyk, and D. Stuckler. 2019. The second information revolution: Digitalization brings opportunities and concerns for public health. European Journal of Public Health 29(3): 3-6.
Miner, A.S., L. Larango, and A.B. Kocaballi. 2020. Chatbots in the fight against the COVID-19 pandemic. NPJ Digital Medicine 3: 65.

Olano, G. 2020. Ping An's audio screening tool helps identify over 1,600 COVID-19 cases. Insurance Business Asia, March 26. https://www.insurancebusinessmag.com/asia/news/breakingnews/ping-ans-audio-screening-tool-helps-identify-over-1600covid19-cases-217968.aspx. Accessed May 1, 2020.

Ricciardi, W., P.P. Barros, A. Bourek, W. Brouwer, T. Kelsey, and L. Lehtonen. 2019. How to govern the digital transformation of health services. European Journal of Public Health 29(3): $7-12$.

Sharwood, S. 2020. Hong Kong coronavirus quarantine evaders collared by cops with the help of smartphone-tracking tech. The Register, March 25. https://www.theregister.co.uk/2020 /03/25/hong_kong_coronavirus/. Accessed June 30, 2020.

United Nations Children's Fund (UNICEF). 2020. Digital contact tracing and surveillance during COVID-19-General and child-specific ethical issues, Innocenti Working Paper 202001, UNICEF Office of Research - Innocenti, Florence. https://www.unicef-irc.org/publications/pdf/WP2020-01.pdf. Accessed June 30, 2020.

World Health Organization. 2017. WHO guidelines on ethical issues in public health surveillance. Geneva: World Health Organization.

-2020. Ethical considerations to guide the use of digital proximity tracking technologies for COVID-19 contact tracing. Geneva: World Health Organization.

Yang, Y. 2020. Zoom faces fire over technology that lets China censor individual users. Financial Times, June 13-14, at 1.

Yau, E. 2020. Singaporean company's new tech to police social distancing will raise alarm when people are too close. South China Morning Post, June 16. https://www.scmp. com/lifestyle/health-wellness/article/3089201/singaporeancompanys-new-tech-police-social-distancing. Accessed June 30, 2020.

Publisher's note Springer Nature remains neutral with regard to jurisdictional claims in published maps and institutional affiliations. 